OPEN ACCESS

Edited by:

Peter Uhlhaas,

University of Glasgow,

United Kingdom

Reviewed by:

Alessio Fracasso,

Utrecht University, Netherlands

Stephan Heckers,

Vanderbilt University, United States

*Correspondence:

Bernd Hinney

bernd.hinney@unibas.ch

Specialty section:

This article was submitted to Schizophrenia

a section of the journal

Frontiers in Psychiatry

Received: 06 October 2020 Accepted: 14 December 2020 Published: 14 January 2021

Citation:

Hinney B, Walter A, Aghlmandi S, Andreou C and Borgwardt S (2021) Does Hippocampal Volume Predict Transition to Psychosis in a High-Risk

Group? A Meta-Analysis

Front. Psychiatry 11:614659.

doi: 10.3389/fpsyt.2020.614659

\section{Does Hippocampal Volume Predict Transition to Psychosis in a High-Risk Group? A Meta-Analysis}

\author{
Bernd Hinney ${ }^{1 *}$, Anna Walter ${ }^{1}$, Soheila Aghlmandi ${ }^{2}$, Christina Andreou ${ }^{1}$ and \\ Stefan Borgwardt ${ }^{1}$ \\ ${ }^{1}$ Department of Psychiatry (UPK), University of Basel, Basel, Switzerland, ${ }^{2}$ Basel Institute for Clinical Epidemiology and \\ Biostatistics, University Hospital Basel, Basel, Switzerland
}

Schizophrenia has a prodromal phase of several years in most patients, making it possible to identify patients at clinical high risk (CHR) for developing the disorder. So far, these individuals are identified based on clinical criteria alone, and there is no reliable biomarker for predicting the transition to psychosis. It is well-established that reductions in brain volume, especially in the hippocampus, are associated with schizophrenia. Therefore, hippocampal volume may serve as a biomarker for psychosis. Several studies have already investigated hippocampal volume in CHR groups. Based on these studies, the present meta-analysis compares the baseline left and right hippocampal volume of $\mathrm{CHR}$ patients who developed a psychosis with that of $\mathrm{CHR}$ patients without such a transition. Our results show no statistically significant effect of the hippocampal volume on the transition risk for psychosis.

Keywords: schizophrenia, psychosis, hippocampus, neuroimaging, high risk, at-risk mental state

\section{INTRODUCTION}

Schizophrenia is a psychiatric illness that is typically preceded by a long prodromal phase (1). The disease has a significant impact on patients' quality-adjusted life years and an often unsatisfactory response to treatment (2). One of the main goals of psychiatric public health government programs is to establish criteria for early detection of schizophrenia. Such criteria could make it possible to implement various forms of secondary prevention to avoid or delay the onset of schizophrenia (3). A large number of studies have accordingly followed-up clinical high-risk (CHR) groups in order to investigate the factors associated with later development of a psychotic disorder (among them the NAPLS- and the PRONIA-project) (4-8). CHR-status is identified according to specific clinical criteria; the most widely established criteria define high-risk based on either (9-12).

a) "attenuated psychotic symptoms" (ASP) or "brief limited intermittent psychotic symptoms" (BLIPS) (these symptoms are manifestations which are typical for psychotic disorders, but which are either not sufficiently severe or too short to warrant the diagnosis itself)

b) genetic vulnerability for psychotic disorders accompanied by a notable downward shift in an individual's social functioning (measured, for example, by difficulties at work or by the inability to live autonomously).

In practice, criterion $b$ is of little relevance in the actual setup of a concrete CHR-group (13). Thus, the dominance of clinical features further highlights the status of the CHR-group as a representation of the prodromal phase. 
When CHR patients develop a psychotic disorder, this is called a "transition." The probability of transition was initially estimated to be between 35 and $40 \%$ in typical CHR-groups (14), but in recent studies, it was lower (15). This could be because of specific protective effects that arise from a diagnosis of CHR: for example, increased risk-awareness of the included person and support from family members, school officials, or other professionals (16).

The low transition rates in CHR-groups mean that it is not feasible to undertake a prophylactic treatment in all CHR patients, especially if such a treatment encompasses the use of antipsychotic medication with the accompanying side-effects.

To enhance the predictive value of CHR-criteria, it has been hypothesized that the inclusion of neuro-imaging data should be helpful $(3,17-19)$. Schizophrenia is known to be accompanied by volume enlargements in the ventricular regions (especially in the later stages of the disease) (20) and volume reductions in several brain areas, among them the frontal cortex, the amygdala, the parahippocampal gyrus and the hippocampus (21$37)$. It has also been suggested that these changes are progressive during the course of the illness (38). Especially hippocampal volume reductions might not only be a result of the disease, but instead have been suggested to be one of its causative factors (39-42). Such causative relationships might be explained by the immense role of the hippocampus in memory formation $(43,44)$. Hippocampal volume reductions and ensuing memory deficits could lead to false hypothesis-testing in CHR-individuals (caused for example by a functional shift from pattern separation to pattern completion) and this might make the individual more prone to psychosis (45). Should such neuroanatomical processes occur, lower hippocampal volumes (compared to healthy controls) should be observable before the actual onset of schizophrenia, which means during the prodromal phase. Such volumetric changes could be a useful biomarker and supplement the predictive value of risk-assessments based on the clinical criteria mentioned above, thus increasing the specificity of the CHR concept (46).

Several studies have carried out MRI-measurements of the hippocampus on members of CHR-groups, and have then compared the results to a parallel arm of healthy controls (4757). The goal of these studies was to assess whether CHR patients have smaller hippocampal volumes compared to healthy controls at baseline (i.e., at the time of diagnosis of the CHR status) compared to healthy individuals. A selection of those studies has been pooled in a meta-analysis by Walter et al., which did not find any significant differences between CHR patients and controls (58). Following up on this finding, the aim of the present metaanalysis is to compare CHR-patients who made the transition to psychosis during the follow-up interval ("converters") with CHRpatients who did not ("non-converters"). This concept has first been employed in a study by Pantelis et al. (59). In the context of our meta-analysis, this procedure implies ignoring the control group of the investigated studies and retrospectively splitting up the case group (CHR patients) into two groups, defined by their transition status. Cases and controls are thus generated from the same cohort, e.g., the CHR-population. Methodologically, this has the significant benefit of reducing heterogeneity between the groups, which is a frequent challenge in case-control studies. Also, from a clinical viewpoint, it is a meaningful approach to remove a potential "dilution" effect that may have occurred in previous studies when combining converters and non-converters in one group.

\section{MATERIALS AND METHODS \\ Eligibility Criteria}

We included studies in our meta-analysis if they investigated individuals that met CHR-criteria. Studies had to assess CHRstatus according to established criteria and the minimum followup period was to be 12 months, as adequate transition rates can be expected after this time (14). Another important factor for eligibility was the presence of the hippocampal volume as an absolute value and not in relation to other parts of the brain [as in voxel-based measurements (VBM)] because the latter values are not ideal for direct statistical comparison and may be compromised through position changes during image registration $(19,60)$.

We assembled eligibility criteria according to the PRISMA$\mathrm{P}$ guidelines. The following paragraph summarizes the inclusion and exclusion criteria that we applied:

\section{Inclusion criteria:}

1. Risk status established according to international research diagnostic criteria for high clinical risk for psychosis (CAARMS, BSIP, PACE, SIPS/SOPS) or primary symptoms (SPIA)

2. Hippocampal volume obtained through the region of interest (ROI) analysis (manually tracing or automated segmentation) reported separately for members of the transition-group and the non-transition group

3. Availability of mean values $( \pm S D)$ of left and right hippocampal volume

4. Publication in a peer-reviewed journal

5. Follow-up interval of at least 12 months.

\section{Exclusion criteria:}

1. A sample size of $<10$ participants

2. Comorbidity with medical or neurological illnesses in patients

3. Any post-mortem assessments

4. Studies on the chromosome 22q11.2 deletion syndrome.

We registered the study with PROSPERO in February 2019, and our study project was approved in April 2019.

\section{Search Strategy and Selection Process}

We conducted a systematic literature search in the public databases Medline and EMBASE on April 28, 2019. We repeated the search on May 28, 2019. We used the following search terms: “(MRI OR magnetic resonance imaging OR neuroimaging) AND (psychos* OR schizophrenia* AND high-risk OR at-risk mental state OR prodrom*) AND hippocamp*".

We removed duplicates using EndNote and two authors (BH and $\mathrm{AW}$ ) screened the references based on the titles and abstracts. All potentially relevant references were read in 


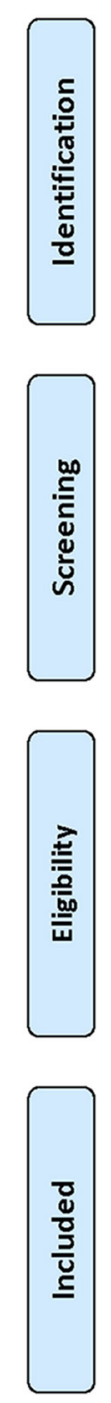

Records identified through

database searching

$(n=157)$

\section{Additional records identified}

through other sources

$(n=2)$

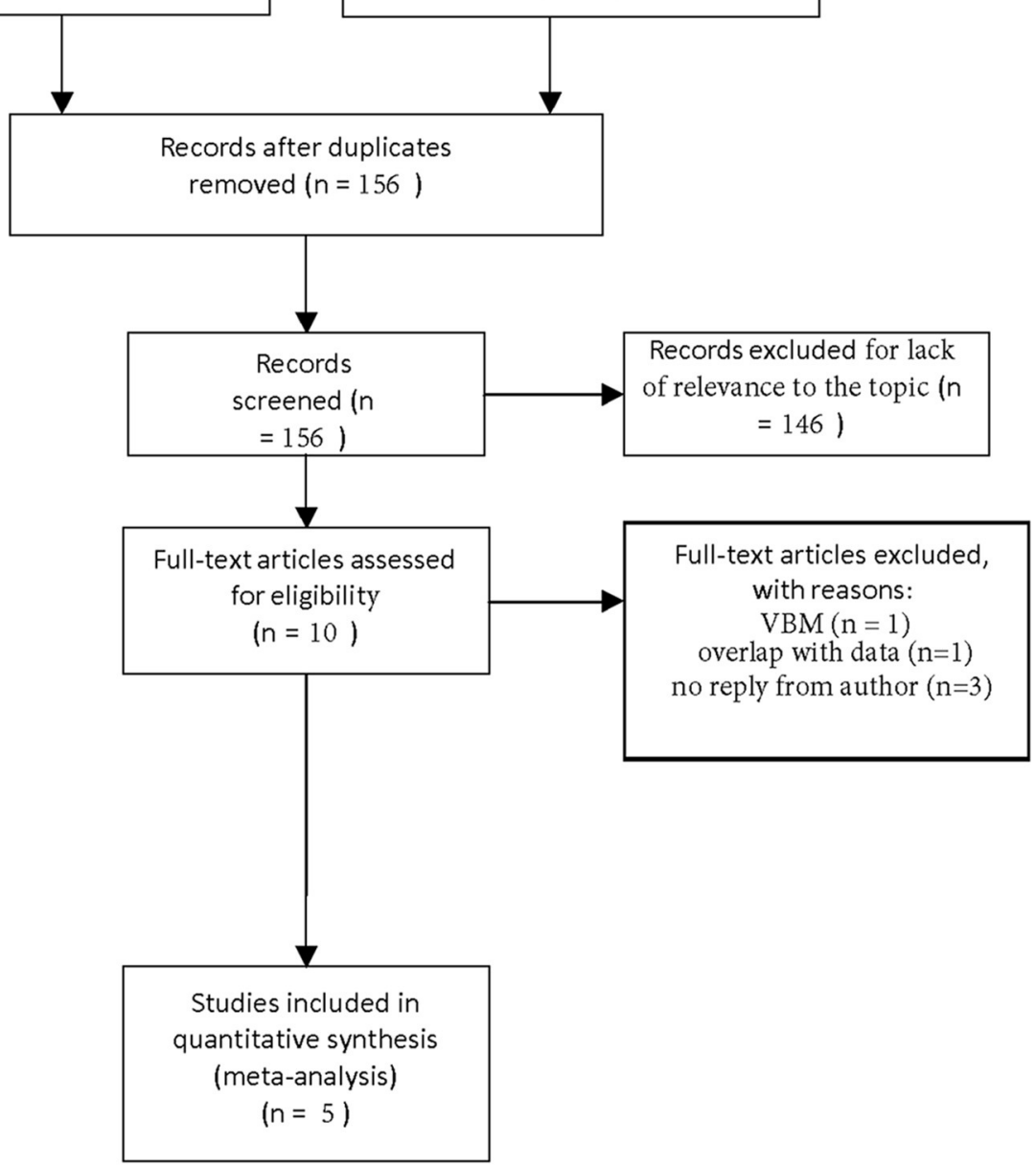

FIGURE 1 | Flow chart of selection process.

full-text and independently assessed by two authors (BH and AW). We resolved any disagreements by consensus. To identify potential additional studies that were not included in electronic databases, we screened the bibliographic references of all included articles.

In this fashion, we recovered 157 papers. Two additional articles were found by screening the references. One hundred and fifty six papers remained after removing duplicates. One hundred and forty six papers could be removed because they were not relevant to our topic or included reprints. Of the 10 remaining articles, we excluded one because it recorded hippocampal volume as a VBM-measurement (61) and we excluded one other study because its sample overlapped with a newer study that better fitted our inclusion criteria (57). In three additional cases, authors did not reply to our request for complete data extraction $(47,54,56)$.
In this process, we could filter out five studies that could be included in our meta-analysis (48, 50, 52, 53, 55). The selection process is visualized in a flow-chart (see Figure 1).

\section{Data Extraction}

Necessary information was extracted by one reviewer $(\mathrm{BH})$ and independently checked by a second reviewer (AW) and entered into a Numbers database. We identified and resolved discrepancies through discussion. Data items to be extracted were: first author name, the cohort, publication date, MRI resolution, hippocampal volume right/left (with standard deviation), number of men/women and mean age of participants (with standard deviation). In some cases, we contacted the authors of the original study in order to provide for the missing variables. 
Hippocampal volume can be presented in two formats: as a "raw," uncorrected number or corrected for the intracranial volume (ICV). There is no consensus in the scientific community which format is more adequate. Hippocampal volume correlates with ICV, but this correlation is not proportional (62). Our goal was to carry out the statistical analysis with both the corrected and the uncorrected values; however, in two cases we could only retrieve the uncorrected values from the authors. All hippocampal volumes stated in this paper are thus the raw volumes in $\mathrm{mm}^{3}$. They represent the mean values of the individual hippocampal measurements in each group (transition/non-transition-group).

\section{Data Synthesis}

We carried out a meta-analysis to assess the differences in the sizes of the left and right hippocampus in a CHR-group for psychosis. Using the data extracted from each paper (see section above), we built an evidence table to investigate possible betweenstudy heterogeneity.

We then used the random-effects model to account for potential statistical heterogeneity, reporting the standardized mean difference and the respective 95\% confidence interval (CI). We used standardized mean differences (SMD) due to the diversity of MRI-devices used (e.g., different resolutions) and applied the Hedges' g method (63). For calculating the pooled SMD using a random-effect model, we used the DersimonianLaird estimator for estimating the between-study variance $\left(\tau^{2}\right)$ (64). Forest plots were generated to show the individual and pooled effect measure, 95\% confidence intervals (CI), the author's name, and study weights for the studies.

We assessed heterogeneity between the results of the studies by using the Cochran's Q test and quantified heterogeneity with the I-squared statistic. Heterogeneity based on the I-square is considered low, moderate, or high when the values are below $25 \%$, between 25 and $75 \%$, or above $75 \%$, respectively.

We investigated sources of inter-study heterogeneity with the univariable random-effects meta-regression analysis that is based on the following primary study characteristics: year of publication, sample size, gender ratio, and MRI resolution. We weighted meta-regression analyses to account for both withinstudy variances of treatment effects and the residual interstudy heterogeneity.
We performed all analyses using the metacont function of the meta package in R 3.6.1 (R Foundation for Statistical Computing, Vienna, Austria) (65).

\section{RESULTS}

\section{Systematic Review}

All five studies that we could include in our meta-analysis were designed as case-control studies in which a healthy control group was compared to a CHR-group for psychosis in terms of baseline volumetric MRI-measurements. After baseline, the CHR-group was clinically followed up to determine transition status. As described above, we ignored the control-group in our analyses and only focused on the CHR-group.

In the following systematic review, we summarize and analyze the five studies regarding the way they established data for CHRgroups and regarding further study characteristics. We do not report special inclusion or exclusion criteria that are already understood due to the requirements we set for our meta-analysis (see section "Materials and Methods").

Dean et al. established CHR-status in an US-American sample using the "Structured Interview for Prodromal Syndromes" (SIPS) (48). Patients who had received antipsychotic medication before the interview were excluded, as were those with a lifelong drug dependence. Two members of the CHR-group who received antipsychotic medication between inclusion and follow-up (12 months later) were also excluded. This is noteworthy, as this may have underweighted the transition group. Reported dropout rates in the CHR-group were low, with $16.7 \%$. The study

TABLE 2A | Summary of group-diffferences between transition-group and non-transition group for each study (right hippocampus).

\begin{tabular}{lccc}
\hline Author & $\begin{array}{c}\text { Standardized } \\
\text { mean difference }\end{array}$ & $\begin{array}{c}\text { 95\% confidence } \\
\text { interval }\end{array}$ & Weight (\%) \\
\hline Dean & 0.7885 & {$[-0.4054 ; 1.9823]$} & 4.5 \\
Pruessner & -0.0062 & {$[-1.0716 ; 1.0592]$} & 5.7 \\
Buehlmann & 0.0761 & {$[-0.5746 ; 0.7268]$} & 14.4 \\
Cannon & -0.0189 & {$[-0.3027 ; 0.2648]$} & 55.6 \\
Harrisberger & -0.475 & {$[-1.0193 ; 0.0693]$} & 19.8 \\
\hline
\end{tabular}

"Weight" refers to the statistical weight within the random effects model.

TABLE 1 | Evidence table.

\begin{tabular}{|c|c|c|c|c|c|c|c|c|c|c|}
\hline \multirow[b]{2}{*}{ Author } & \multirow[b]{2}{*}{ Year } & \multirow[b]{2}{*}{ Cohort } & \multicolumn{4}{|c|}{ Transition group } & \multicolumn{4}{|c|}{ Non-transition group } \\
\hline & & & $N$ men & $N$ women & Mean age & SD age & $N$ men & $N$ women & Mean age & SD age \\
\hline Dean & 2016 & Boulder & 2 & 1 & 19.33 & 2.08 & 20 & 15 & 18.89 & 1.39 \\
\hline Pruessner & 2017 & Montreal & 2 & 2 & 21.14 & 5.1 & 11 & 11 & 19.97 & 3.15 \\
\hline Buehlmann & 2010 & Basel & 11 & 5 & 26.4 & 6.5 & 11 & 10 & 23.4 & 6 \\
\hline Cannon & 2015 & NAPLS & 36 & 19 & 19.18 & 3.77 & 213 & 147 & 19.78 & 4.23 \\
\hline Harrisberger & 2016 & Basel and Zurich & 9 & 7 & 25.56 & 7.31 & 50 & 25 & 23.22 & 4.35 \\
\hline
\end{tabular}




\begin{tabular}{|c|c|c|c|c|c|c|c|c|}
\hline \multirow[b]{2}{*}{ Study } & \multirow[b]{2}{*}{ Total } & Experimental & \multirow[b]{2}{*}{ Total } & Control & Standardised Mean & \multirow[b]{2}{*}{ SMD } & \multirow[b]{2}{*}{$95 \%-\mathrm{Cl}$} & \multirow[b]{2}{*}{ Weight } \\
\hline & & Mean SD & & Mean $\quad$ SD & Difference & & & \\
\hline DEAN & 3 & 4648.97469 .2100 & 35 & 4332.45388 .0700 & & 0.81 & {$[-0.39 ; 2.00]$} & $4.8 \%$ \\
\hline BRUELMANN & 16 & 3194.30215 .5000 & 21 & 3165.80447 .4000 & & 0.08 & {$[-0.57 ; 0.73]$} & $14.9 \%$ \\
\hline CANNON & 55 & 4237.84378 .9256 & 360 & 4246.77483 .1807 & & -0.02 & {$[-0.30 ; 0.26]$} & $53.9 \%$ \\
\hline HARRISBERGER & 16 & 3913.29469 .8045 & 75 & 4201.09624 .0019 & +4 & -0.48 & {$[-1.02 ; 0.07]$} & $20.4 \%$ \\
\hline \multirow{2}{*}{\multicolumn{3}{|c|}{$\begin{array}{l}\text { Random effects model } \\
\text { Prediction interval } \\
\text { Heterogeneity: } I^{2}=12 \%, \tau^{2}=0.0133, p=0.34\end{array}$}} & & & & \multirow{2}{*}{\multicolumn{2}{|c|}{$\begin{array}{r}-0.06[-0.32 ; 0.21] \\
{[-0.62 ; 0.51]}\end{array}$}} & \multirow{2}{*}{$100.0 \%$} \\
\hline & & & & & -1 & & & \\
\hline
\end{tabular}

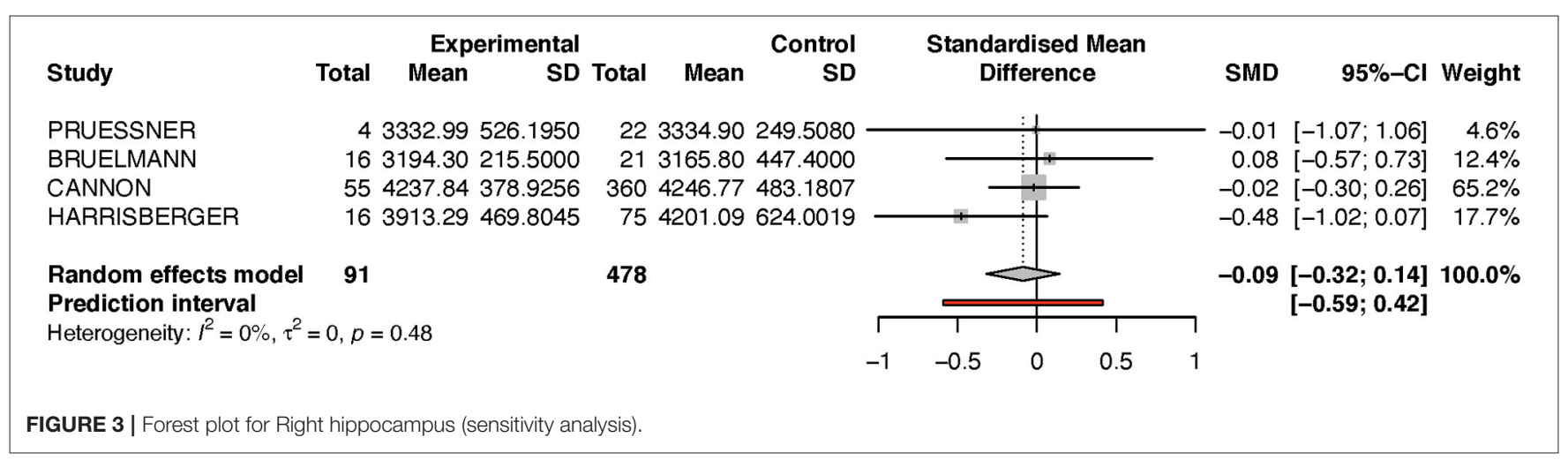

had a rather short follow-up period of 12 months, which can be assumed to have only captured a part of the actual scope of transition. Scanning was done on a 3-Tesla device and automated segmentation with FreeSurfer (version 5.3.0) was used for image processing.

Cannon et al. also used the SIPS for CHR-inclusion in a US-American sample. There were no special exclusion criteria for the use of antipsychotic medication or drug abuse, as the study was done in a naturalistic setting. Drop-out rates were not reported, and the follow-up periods were short with 12 months (52). Scanning was performed on a 3-Tesla device and automated segmentation with FreeSurfer (version 5.2.0) was used for image processing.

Pruessner et al. used the "Comprehensive Assessment of At Risk Mental States" (CAARMS)-interview to establish CHRstatus in a Canadian sample. Anyone with a history of substance use disorder or mental illness was excluded. Drop-out rates were not reported, and the regular follow-up interval was 2 years (55). Scanning was performed on a 1,5-Tesla device and automated segmentation with a self-designed tool (66) was used for image processing.

Harrisberger et al. used the "Basel Screening Instrument for Psychoses" (BSIP) in a Swiss sample to establish CHR status (53). Only antipsychotic-naive individuals were included in the CHR group. Drop-out was not reported, and the follow-up interval was 3 years. Scanning was performed on a 3-Tesla device and automated segmentation with the software FSL-FIRST (67) was used for image processing.
TABLE 2B | Summary of group-diffferences between transition-group and non-transition group for each study (left hippocampus).

\begin{tabular}{lccc}
\hline Author & $\begin{array}{c}\text { Standardized } \\
\text { mean difference }\end{array}$ & $\begin{array}{c}\text { 95\% confidence } \\
\text { interval }\end{array}$ & Weight (\%) \\
\hline Dean & 1.2041 & {$[-0.0092 ; 2.4174]$} & 9.5 \\
Pruessner & -0.4011 & {$[-1.4730 ; 0.6708]$} & 11.4 \\
Buehlmann & 0.2221 & {$[-0.4305 ; 0.8747]$} & 20.7 \\
Cannon & 0.0975 & {$[-0.1863 ; 0.3814]$} & 34.1 \\
Harrisberger & -0.5401 & {$[-1.0858 ; 0.0056]$} & 24.3
\end{tabular}

"Weight" refers to the statistical weight within the random effects model.

Buehlmann et al. used the "Personal Assessment and Crisis Evaluation" (PACE)-criteria for CHR-status in a Swiss sample (50) (there was no overlap with the study by Harrisberger et al.). The study was naturalistic and did not require any specific inclusion or exclusion criteria beyond those generally required for inclusion in our meta-analysis. Drop-out rates were not reported, and the follow-up interval was 3 years. Scanning was performed on a 1,5-Tesla device and manual tracing combined with AMIRA was used for image processing.

\section{Participant Characteristics}

The transition group (case group) consisted of 94 members (60 men, 34 women). The mean age was 21.57 . The standard 


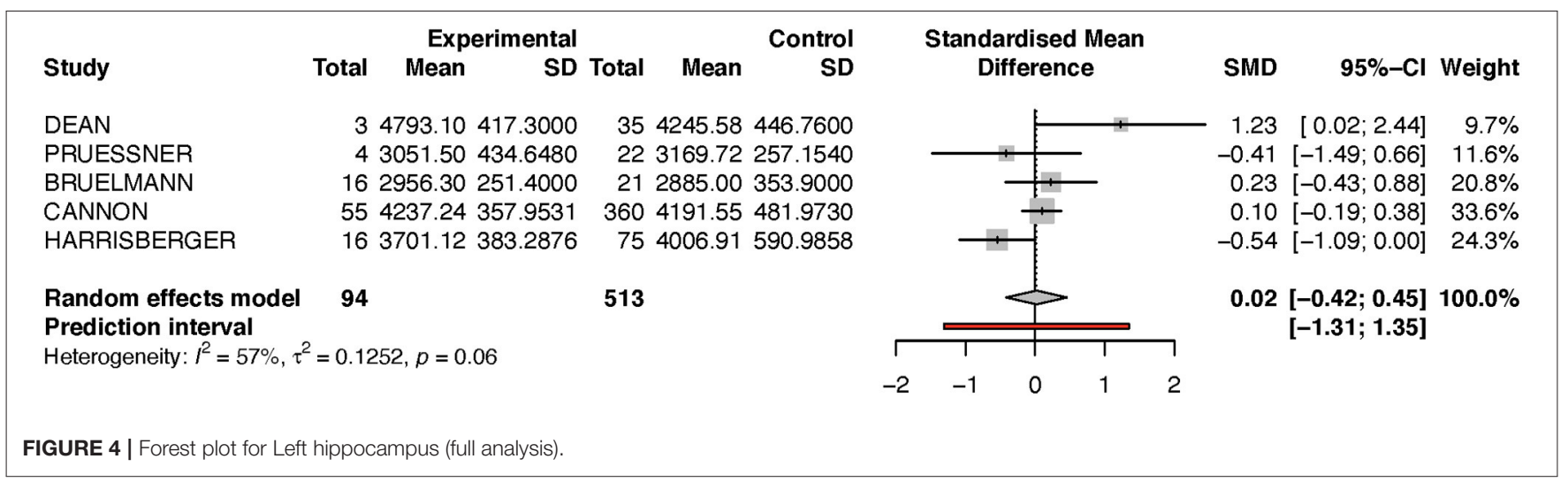

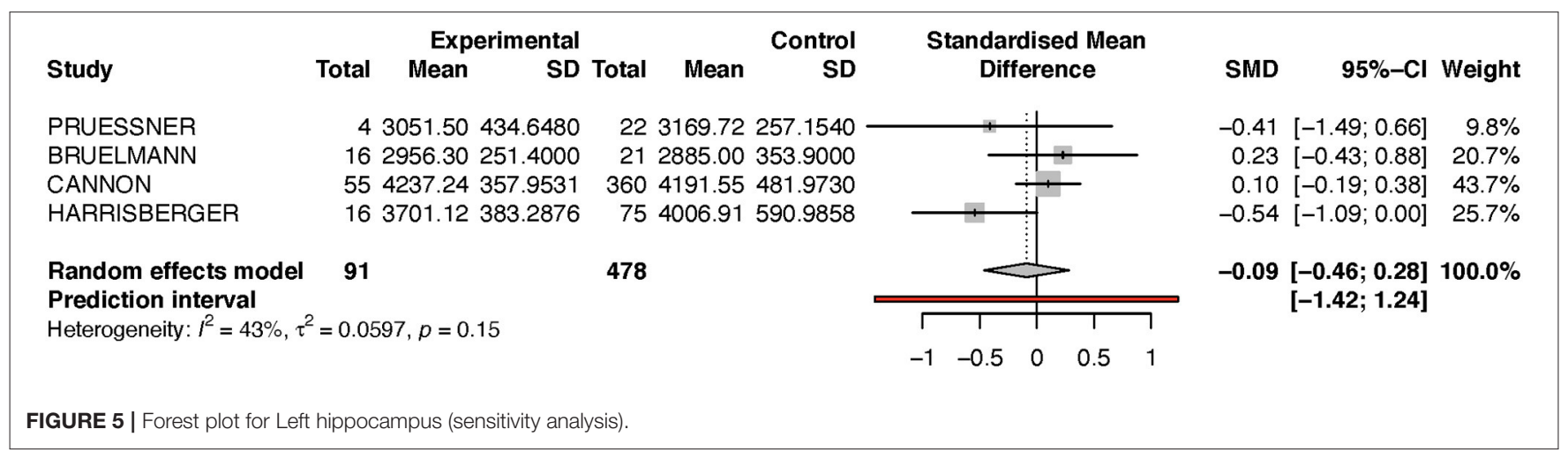

TABLE 3 | Univariable meta-regression including all studies.

\begin{tabular}{|c|c|c|c|c|c|c|}
\hline & \multicolumn{3}{|c|}{ Right hippocampus } & \multicolumn{3}{|c|}{ Left hippocampus } \\
\hline & Estimate & $95 \% \mathrm{Cl}$ & $p$-value & Estimate & $95 \% \mathrm{Cl}$ & $p$-value \\
\hline Publication year (change per year) & -0.036 & $(-0.245,0.174)$ & 0.625 & -0.065 & $(-0.382,0.253)$ & 0.562 \\
\hline Sample size (change per N) & -0.0001 & $(-0.004,0.003)$ & 0.943 & -0.00001 & $(-0.006,0.006)$ & 0.982 \\
\hline Gender ration & 0.138 & $(-1.620,1.896)$ & 0.818 & 0.194 & $(-2.613,3.001)$ & 0.84 \\
\hline MRI resolution & 0.228 & $(-0.421,0.878)$ & 0.345 & 0.315 & $(-0.835,1.464)$ & 0.448 \\
\hline
\end{tabular}

deviation of age (transition group) in the individual studies ranged from 2 to 7 years (see Table 1 for details).

The non-transition group (control group) consisted of 513 members (305 men, 208 women). The mean age was 20.38 . The standard deviation of age in the individual studies ranged from 1 to 4 years (see Table 1 for details).

\section{Results of Meta-Analysis}

Due to the observed heterogeneity between the studies, we only report the results for the random-effects meta-analysis.

Looking at the right hippocampus, the standard mean difference shows that the volume of the right hippocampus is $6 \%$ less in the transition group than in the non-transition group (see Table 2A and Figure 2). The heterogeneity $\left(I^{2}\right)$ between the studies is $12 \%$, which indicates low heterogeneity.

Because the study of Dean et al. had a very small sample size, and because its results diverged from that of the other studies, we performed a sensitivity analysis by excluding this one study (see Figure 3). Performing this analysis reduced heterogeneity to zero, and moved the $p$-value closer to the direction of rejection of the null-hypothesis. Nevertheless, in both analyses, the results were not statistically significant and indicate at most a numerical trend.

For the left hippocampus, the heterogeneity $\left(I^{2}\right)$ was much larger, with 57\% (see Table $2 \mathbf{B}$ and Figure 4). The results have thus to be interpreted with caution, as is also indicated by the wide predictive interval. Initial analysis showed the left hippocampal volume to be $2 \%$ larger in the transition group. This is in contradiction to our hypothesis that the transition group has a smaller left hippocampus at baseline. However, when the sensitivity analysis was performed (again excluding the Dean et al. study, see Figure 5), this result was reversed, and the transition group's hippocampal volume was $9 \%$ smaller compared to the non-transition group. The results of both analyses for the left hippocampus are not statistically significant. 


\section{Results of Meta-Regression}

We performed a meta-regression for the following variables: year of publication, sample size, gender ratio and resolution of MRI-device (see Table 3). None of the assessed variables in the meta-regression significantly contributed to the betweenstudy heterogeneity.

\section{DISCUSSION}

Our meta-analysis investigated whether hippocampal volume at baseline predicts the transition to psychosis in a CHR-group. Our study did not find a statistically significant relationship between these parameters. However, we did find a trend that points into such a direction when looking at the right hippocampus. This trend was further confirmed after performing a sensitivity analysis. The investigated studies showed much more heterogeneous results for the left hippocampus. Interestingly, a study by Seidman investigating the parahippocampal gyrus as a vulnerability factor for schizophrenia also found more pronounced effects for the right side of the brain (37).

It remains to be seen if future studies can corroborate this trend. If so, it will be interesting to see whether such a result is bilateral or whether the unilateral quality (e.g., the prominence of changes in the right hippocampus) still holds true.

Looking at the results of the systematic review, the most rigorous study-protocol was provided by two studies that excluded a history of mental illness and of drug abuse. Thus, the most important potential confounders regarding hippocampal volume were removed. Two other studies excluded either one or the other of those preconditions. Very few studies reported drop-out rates in the CHR-group, and follow-up intervals varied considerably. Transition rates are generally assumed to be $18 \%$ after a follow-up of 6 months, 22\% after 1 year, 29\% after 2 years, and $36 \%$ after 3 years (14). Thus, only two of the five studies we analyzed employed the upper limit regarding follow-up.

\section{Possible Clinical Implications}

There is an ongoing debate about the concrete implications of risk predictions for members of CHR-groups. For a summary of the most important points in question, see Andreou and Borgwardt (19). For hippocampal volume to be of clinical use, a good cut-off margin between likely converters and nonconverters will be required. This could provide clinicians with a biomarker that can be used to differentiate the CHR-group into an "extreme" risk group and a "reduced" risk group. Prophylactic treatment efforts could then be focused on those individuals who will most likely benefit from them.

\section{Limitations and Future Directions}

Certain limitations of our meta-analysis need to be discussed. First of all, the overall number of studies was rather small. Second, looking only at the hippocampal areas may underestimate the complexity of the neuronal network that underlies the development of paranoid schizophrenia, and in addition McHugo recently noted that it may not be the whole hippocampus that changes in volume before and during psychosis, but rather specific subfields (especially the anterior hippocampus) (68). More precisely, it might be necessary to not only test left-right-asymmetry between global hippocampus volume (as was done in our study), but also to conduct a differential regional analysis (e.g., anterior or posterior region of the hippocampus) or a subfield analysis (e.g., subiculum, cornu ammonis, dentate gyrus).

Thirdly, follow-up periods varied between the studies, and short follow-up times may have led to some falsenegatives within the non-transition-group. This would reduce the likelihood of discovering any significant relationship between (premorbid) hippocampal brain volume and the likelihood of developing psychosis. All of these factors could modify a relevant relationship between hippocampal volume and transition risk.

Methodologically, two of our studies scanned the brains of study participants on a 1.5 Tesla device while the other three used 3 Tesla devices. It is possible that the resulting differences in resolution affected the accuracy of the measurement of the hippocampus, although there generally seems to be a high correlation between 1.5 and 3 Tesla measurements of hippocampal volume $(69,70)$. Similarly, while four out of the five studies used differing versions of automated segmentation with FreeSurfer or other Software for the measurements of the hippocampal volume, the paper by Buehlmann et al. used manual segmentation. Regarding the image processing, the MRIsequences used (T1 or T2) might also have an impact on the volumetric measurements, but this was a data point that was not available to us. It must also be noted that correction of raw hippocampal volume for ICV might have had a significant effect on the outcome.

Finally, none of the papers we analyzed explicitly mentioned the diagnostic instruments used to establish transition. While it can be assumed that transition to psychosis was usually confirmed by applying the ICD-10 and/or DSM-IV criteria for schizophrenia through an experienced clinician, it should be noted that ICD-10 and DMS-IV criteria for schizophrenia do differ slightly and that "transition to psychosis" is not in every case equivalent with the development of schizophrenia. Velakoulis et al. have noted that hippocampal volume loss may be much less pronounced in diagnoses of the schizophreniform spectrum than in schizophrenia itself (56). The diagnostic instruments used to establish CHR-status (CAARMS, BSIP, PACE, SIPS) also varied. Taken together, there are a number of potential sources of heterogeneity that should be considered when conducting further studies on the topic.

On a more general level, the concept of a macroscopically visible biomarker (e.g., shrinkages of the hippocampus in volumetric MRI-measurements) may not capture the reality of the prodromal phase. It is quite possible that during the prodromal phase, it is not the volume of a whole-brain structure that changes, but rather the way neuronal populations communicate with each other. Other techniques would have to be adopted to capture such changes, for example, MRT-assessments of functional connectivity as done by Blessing et al. (71). Also, volumetric assessments can be enhanced by measurements of cortical thickness, surface area, and gyrification to capture a wider range of potential changes in brains of individuals who go on to develop a psychosis (19). It is also possible to determine 
hippocampal volume at different time points (as was done by Cannon et al.) to determine a rate of change during the prodromal phase.

\section{Implications for Members of CHR-Groups Who Are Non-converters}

An important question is whether CHR-members in the nontransition group can be regarded as healthy individuals or whether they constitute a subclinical set of the psychotic spectrum. While some of those individuals will eventually make the transition to psychosis (albeit after the follow-up interval), there will be a significant proportion of non-converters. For this group of individuals, essentially two etiological scenarios are possible:

a) non-converters carry the same risk for development of a psychosis as the members of the transition-group, but due to favorable circumstances (for example a very healthy life-style) psychosis does not manifest. This would be in accordance with the diathesis-stress-model of schizophrenia (72).

b) non-converters do not carry a relevantly heightened risk for the development of a psychosis. They are thus "wrongfully" included in the CHR-set due to the intentionally low specificity-levels of criteria used in the establishment of risk-groups.

Our study cannot answer the question which of these scenarios is most likely. It would be beneficial to include the subgroup mentioned in a long-term follow-up to gain more statistical information on their outcome and CHR-members should also be evaluated according to the healthiness of the lifestyle they adopt.

\section{REFERENCES}

1. Hafner H, Riecher-Rossler A, Hambrecht M, Maurer K, Meissner S, Schmidtke A, et al. IRAOS: an instrument for the assessment of onset and early course of schizophrenia. Schizophr Res. (1992) 6:209-23. doi: 10.1016/0920-9964(92)90004-O

2. Saha S, Chant D, Welham J, McGrath J. A systematic review of the prevalence of schizophrenia. PLoS Med. (2005) 2:e141. doi: 10.1371/journal.pmed.0020141

3. DeLisi LE. The concept of progressive brain change in schizophrenia: implications for understanding schizophrenia. Schizophr Bull. (2008) 34:31221. doi: $10.1093 / \mathrm{schbul} / \mathrm{sbm} 164$

4. Riecher-Rossler A, Gschwandtner U, Aston J, Borgwardt S, Drewe M, Fuhr P, et al. The basel early-detection-of-psychosis (FEPSY)-studydesign and preliminary results. Acta Psychiatr Scand. (2007) 115:114-25. doi: 10.1111/j.1600-0447.2006.00854.x

5. Yung AR, McGorry PD, McFarlane CA, Jackson HJ, Patton GC, Rakkar A. Monitoring and care of young people at incipient risk of psychosis. Schizophr Bull. (1996) 22:283-303. doi: 10.1093/schbul/22.2.283

6. Miller TJ, McGlashan TH, Rosen JL, Cadenhead K, Cannon T, Ventura J, et al. Prodromal assessment with the structured interview for prodromal syndromes and the scale of prodromal symptoms: predictive validity, interrater reliability, and training to reliability. Schizophr Bull. (2003) 29:70315. doi: 10.1093/oxfordjournals.schbul.a007040

7. Koutsouleris N, Kambeitz-Ilankovic L, Ruhrmann S, Rosen M, Ruef A, Dwyer D, et al. Prediction models of functional outcomes for individuals in the clinical high-risk state for psychosis or with recent-onset depression: a multimodal, multisite machine learning analysis. JAMA Psychiatry. (2018) 75:1156-72. doi: 10.1001/jamapsychiatry.2018.2165

\section{Summary}

In line with previous research, our meta-analysis indicates that there occur hippocampal volume changes in members of an ultra-high-risk-group before the transition to psychosis, but results did not reach significance thresholds. Larger samples are needed for future research in this area, and studies should not only look in more detail at the macroscopic level, but also assess the changes that occur on a functional level.

\section{DATA AVAILABILITY STATEMENT}

The original contributions presented in the study are included in the article/supplementary materials, further inquiries can be directed to the corresponding author/s.

\section{AUTHOR CONTRIBUTIONS}

$\mathrm{SB}$ and $\mathrm{AW}$ conceived the concept of the paper. $\mathrm{BH}$ and $\mathrm{AW}$ carried out the analysis of relevant literature. $\mathrm{BH}$ wrote the introduction, the results and the discussion section. $\mathrm{BH}$ and SA carried out the statistical analysis. SA wrote the Material and Methods section. CA revised the final manuscript. All authors contributed to the article and approved the submitted version.

\section{ACKNOWLEDGMENTS}

The authors acknowledge Yoonho Chan, Derek Dean, and Marita Pruessner who provided additional information and volumetric data.

8. Addington J, Liu L, Buchy L, Cadenhead K, Cannon T, Cornblatt B, et al. North American Prodrome Longitudinal Study (NAPLS 2): the prodromal symptoms. J Nervous Mental Dis. (2015) 203:328-35. doi: 10.1097/NMD.0000000000000290

9. Riecher-Rössler A, Aston J, Ventura J, Merlo M, Borgwardt S, Gschwandtner U, et al. Das Basel Screening Instrument für Psychosen (BSIP) : entwicklung, aufbau, reliabilität und validität. Fortschr Neurol Psychiatr. (2008) 76:207-16. doi: 10.1037/t68527-000

10. Fusar-Poli P, Cappucciati M, Rutigliano G, Lee TY, Beverly Q, Bonoldi I, et al. Towards a standard psychometric diagnostic interview for subjects at ultra high risk of psychosis: CAARMS versus SIPS. Psychiatry J. (2016) 2016:7146341. doi: 10.1155/2016/7146341

11. Woods SW, Walsh BC, Addington J, Cadenhead KS, Cannon TD, Cornblatt $\mathrm{BA}$, et al. Current status specifiers for patients at clinical high risk for psychosis. Schizophr Res. (2014) 158:69-75. doi: 10.1016/j.schres.2014. 06.022

12. Andreou C, Bailey B, Borgwardt S. Assessment and treatment of individuals at high risk for psychosis. BJPsych Adv. (2019) 25:177-84. doi: 10.1192/bja.2019.3

13. Schultze-Lutter F, Michel C, Schmidt SJ, Schimmelmann BG, Maric NP, Salokangas RK, et al. EPA guidance on the early detection of clinical high risk states of psychoses. Eur Psychiatry. (2015) 30:405-16. doi: 10.1016/j.eurpsy.2015.01.010

14. Fusar-Poli P, Bonoldi I, Yung AR, Borgwardt S, Kempton MJ, Valmaggia L, et al. Predicting psychosis: meta-analysis of transition outcomes in individuals at high clinical risk. Arch Gen Psychiatry. (2012) 69:220-9. doi: 10.1001/archgenpsychiatry.2011.1472

15. Wiltink S, Velthorst E, Nelson B, McGorry PM, Yung AR. Declining transition rates to psychosis: the contribution of potential changes in referral 
pathways to an ultra-high-risk service. Early Interv Psychiatry. (2015) 9:200-6. doi: 10.1111/eip.12105

16. Raballo A, Poletti M, Carpenter WT. Rethinking the psychosis threshold in clinical high risk. Schizophr Bull. (2019) 45:1-2. doi: 10.1093/schbul/sby149

17. Gothelf D, Soreni N, Nachman RP, Tyano S, Hiss Y, Reiner O, et al. Evidence for the involvement of the hippocampus in the pathophysiology of schizophrenia. Eur Neuropsychopharmacol. (2000) 10:389-95. doi: 10.1016/S0924-977X(00)00097-3

18. Heckers S, Konradi C. Hippocampal neurons in schizophrenia. J Neural Transm. (2002) 109:891-905. doi: 10.1007/s007020200073

19. Andreou C, Borgwardt S. Structural and functional imaging markers for susceptibility to psychosis. Mol Psychiatry. (2020) 25:2773-85. doi: 10.1038/s41380-020-0679-7

20. Berger GE, Bartholomeusz CF, Wood SJ, Ang A, Phillips LJ, Proffitt T, et al. Ventricular volumes across stages of schizophrenia and other psychoses. Aust N Z J Psychiatry. (2017) 51:1041-51. doi: 10.1177/0004867417715914

21. Nenadic I, Dietzek M, Schonfeld N, Lorenz C, Gussew A, Reichenbach JR, et al. Brain structure in people at ultra-high risk of psychosis, patients with first-episode schizophrenia, and healthy controls: a VBM study. Schizophr Res. (2015) 161:169-76. doi: 10.1016/j.schres.2014.10.041

22. Witthaus H, Mendes U, Brune M, Ozgurdal S, Bohner G, Gudlowski Y, et al. Hippocampal subdivision and amygdalar volumes in patients in an atrisk mental state for schizophrenia. J Psychiatry Neurosci. (2010) 35:33-40. doi: $10.1503 /$ jpn. 090013

23. Haijma SV, Van Haren N, Cahn W, Koolschijn P, Hulshoff Pol H, Kahn R. Brain volumes in schizophrenia: a meta-analysis in over 18000 subjects. Schizophr Bull. (2013) 39:1129-38. doi: 10.1093/schbul/sbs118

24. Okada N, Fukunaga M, Yamashita F, Koshiyama D, Yamamori H, Ohi K, et al. Abnormal asymmetries in subcortical brain volume in schizophrenia. Mol Psychiatry. (2016) 21:1460-6. doi: 10.1038/mp.2015.209

25. Sui J, Pearlson GD, Du Y, Yu Q, Jones TR, Chen J, et al. In search of multimodal neuroimaging biomarkers of cognitive deficits in schizophrenia. Biol Psychiatry. (2015) 78:794-804. doi: 10.1016/j.biopsych.2015.02.017

26. Cahn W, Hulshoff Pol HE, Bongers M, Schnack HG, Mandl RC, Van Haren NE, et al. Brain morphology in antipsychotic-naive schizophrenia: a study of multiple brain structures. Br J Psychiatry Suppl. (2002) 43:s66-72. doi: 10.1192/bjp.181.43.s66

27. Steen RG, Mull C, McClure R, Hamer RM, Lieberman JA. Brain volume in first-episode schizophrenia: systematic review and meta-analysis of magnetic resonance imaging studies. Br J Psychiatry. (2006) 188:510-8. doi: 10.1192/bjp.188.6.510

28. Jung WH, Borgwardt S, Fusar-Poli P, Kwon JS. Gray matter volumetric abnormalities associated with the onset of psychosis. Front Psychiatry. (2012) 3:101. doi: 10.3389/fpsyt.2012.00101

29. Ziermans TB, Schothorst PF, Schnack HG, Koolschijn PC, Kahn RS, van Engeland H, et al. Progressive structural brain changes during development of psychosis. Schizophr Bull. (2012) 38:519-30. doi: 10.1093/schbul/ sbq113

30. Ebdrup BH, Glenthoj B, Rasmussen H, Aggernaes B, Langkilde AR, Paulson $\mathrm{OB}$, et al. Hippocampal and caudate volume reductions in antipsychoticnaive first-episode schizophrenia. J Psychiatry Neurosci. (2010) 35:95-104. doi: $10.1503 /$ jpn.090049

31. Bois C, Levita L, Ripp I, Owens DC, Johnstone EC, Whalley HC, et al. Hippocampal, amygdala and nucleus accumbens volume in first-episode schizophrenia patients and individuals at high familial risk: a cross-sectional comparison. Schizophr Res. (2015) 165:45-51. doi: 10.1016/j.schres.2015.03.024

32. Borgwardt SJ, McGuire PK, Aston J, Gschwandtner U, Pfluger MO, Stieglitz RD, et al. Reductions in frontal, temporal and parietal volume associated with the onset of psychosis. Schizophr Res. (2008) 106:108-14. doi: $10.1016 /$ j.schres.2008.08.007

33. González-Vivas C, Soldevila-Matías P, Sparano O, García-Martí G, MartíBonmatí L, Crespo-Facorro B, et al. Longitudinal studies of functional magnetic resonance imaging in first-episode psychosis: a systematic review. Eur Psychiatry. (2019) 59:60-9. doi: 10.1016/j.eurpsy.2019. 04.009

34. Li W, Li K, Guan P, Chen Y, Xiao Y, Lui S, et al. Volume alteration of hippocampal subfields in first-episode antipsychotic-naïve schizophrenia patients before and after acute antipsychotic treatment. Neuroimage Clin. (2018) 20:169-76. doi: 10.1016/j.nicl.2018.07.008

35. Baglivo V, Cao B, Mwangi B, Bellani M, Perlini C, Lasalvia A, et al. Hippocampal subfield volumes in patients with first-episode psychosis. Schizophr Bull. (2018) 44:552-9. doi: 10.1093/schbul/sbx108

36. Haukvik U, Tamnes C, Söderman E, Agartz I. Neuroimaging hippocampal subfields in schizophrenia and bipolar disorder: a systematic review and meta-analysis. J Psychiatric Res. (2018) 104:217-26. doi: 10.1016/j.jpsychires.2018.08.012

37. Seidman L, Pantelis C, Keshavan M, Faraone S, Goldstein J, Horton N, et al. A review and new report of medial temporal lobe dysfunction as a vulnerability indicator for schizophrenia: a magnetic resonance imaging morphometric family study of the parahippocampal gyrus. Schizophr Bull. (2003) 29:803-30. doi: 10.1093/oxfordjournals.schbul.a007048

38. Lieberman J, Chakos M, Wu H, Alvir J, Hoffman E, Robinson D, et al. Longitudinal study of brain morphology in first episode schizophrenia. Biol Psychiatry. (2001) 49:487-99. doi: 10.1016/S0006-3223(01)01067-8

39. Bernard JA, Orr JM, Mittal VA. Abnormal hippocampal-thalamic white matter tract development and positive symptom course in individuals at ultra-high risk for psychosis. NPJ Schizophr. (2015) 1:15009. doi: $10.1038 /$ npjschz.2015.9

40. Sapolsky RM. Glucocorticoids and hippocampal atrophy in neuropsychiatric disorders. Arch Gen Psychiatry. (2000) 57:925-35. doi: 10.1001/archpsyc.57.10.925

41. Nakahara S, Matsumoto M, van Erp T. Hippocampal subregion abnormalities in schizophrenia: a systematic review of structural and physiological imaging studies. Neuropsychopharmacol Rep. (2018) 38:156-66. doi: $10.1002 / \mathrm{npr} 2.12031$

42. Vargas T, Dean D, Osborne K, Gupta T, Ristanovic I, Ozturk S, et al. Hippocampal subregions across the psychosis spectrum. Schizophr Bull. (2018) 44:1091-9. doi: 10.1093/schbul/sbx160

43. Opitz B. Memory function and the hippocampus. Front Neurol Neurosci. (2014) 34:51-9. doi: 10.1159/000356422

44. Burgess N, Maguire E, O'Keefe J. The human hippocampus and spatial and episodic memory. Neuron. (2002) 35:625-41. doi: 10.1016/S0896-6273(02)00830-9

45. Tamminga CA, Stan AD, Wagner AD. The hippocampal formation in schizophrenia. Am J Psychiatry. (2010) 167:1178-93. doi: 10.1176/appi.ajp.2010.09081187

46. Cannon TD. Brain biomarkers of vulnerability and progression to psychosis. Schizophr Bull. (2016) 42(Suppl. 1):S127-32. doi: 10.1093/schbul/sbv173

47. Wood SJ, Kennedy D, Phillips LJ, Seal ML, Yucel M, Nelson B, et al. Hippocampal pathology in individuals at ultra-high risk for psychosis: a multi-modal magnetic resonance study. Neuroimage. (2010) 52:62-8. doi: 10.1016/j.neuroimage.2010.04.012

48. Dean DJ, Orr JM, Bernard JA, Gupta T, Pelletier-Baldelli A, Carol EE, et al. Hippocampal shape abnormalities predict symptom progression in neuroleptic-free youth at ultrahigh risk for psychosis. Schizophr Bull. (2016) 42:161-9. doi: 10.1093/schbul/sbv086

49. Walter A, Studerus E, Smieskova R, Kuster P, Aston J, Lang UE, et al. Hippocampal volume in subjects at high risk of psychosis: a longitudinal MRI study. Schizophr Res. (2012) 142:217-22. doi: 10.1016/j.schres.2012.10.013

50. Buehlmann E, Berger GE, Aston J, Gschwandtner U, Pflueger MO, Borgwardt SJ, et al. Hippocampus abnormalities in at risk mental states for psychosis? A cross-sectional high resolution region of interest magnetic resonance imaging study. J Psychiatr Res. (2010) 44:447-53. doi: 10.1016/j.jpsychires.2009.10.008

51. Phillips LJ, Velakoulis D, Pantelis C, Wood S, Yuen HP, Yung AR, et al. Nonreduction in hippocampal volume is associated with higher risk of psychosis. Schizophr Res. (2002) 58:145-58. doi: 10.1016/S0920-9964(01)00392-9

52. Cannon TD, Chung Y, He G, Sun D, Jacobson A, van Erp TG, et al. Progressive reduction in cortical thickness as psychosis develops: a multisite longitudinal neuroimaging study of youth at elevated clinical risk. Biol Psychiatry. (2015) 77:147-57. doi: 10.1016/j.biopsych.2014.05.023

53. Harrisberger F, Buechler R, Smieskova R, Lenz C, Walter A, Egloff L, et al. Alterations in the hippocampus and thalamus in individuals at high risk for psychosis. NPJ Schizophr. (2016) 2:16033. doi: 10.1038/npjschz.2016.33

54. Klauser P, Zhou J, Lim JK, Poh JS, Zheng H, Tng HY, et al. Lack of evidence for regional brain volume or cortical thickness abnormalities in youths at clinical 
high risk for psychosis: findings from the longitudinal youth at risk study. Schizophr Bull. (2015) 41:1285-93. doi: 10.1093/schbul/sbv012

55. Pruessner M, Bechard-Evans L, Pira S, Joober R, Collins DL, Pruessner JC, et al. Interplay of hippocampal volume and hypothalamus-pituitary-adrenal axis function as markers of stress vulnerability in men at ultra-high risk for psychosis. Psychol Med. (2017) 47:471-83. doi: 10.1017/S0033291716002658

56. Velakoulis D, Wood SJ, Wong MT, McGorry PD, Yung A, Phillips L, et al. Hippocampal and amygdala volumes according to psychosis stage and diagnosis: a magnetic resonance imaging study of chronic schizophrenia, first-episode psychosis, and ultra-high-risk individuals. Arch Gen Psychiatry. (2006) 63:139-49. doi: 10.1001/archpsyc.63.2.139

57. Mittal VA, Gupta T, Orr JM, Pelletier-Baldelli A, Dean DJ, LunsfordAvery JR, et al. Physical activity level and medial temporal health in youth at ultra high-risk for psychosis. J Abnorm Psychol. (2013) 122:1101-10. doi: $10.1037 / \mathrm{a} 0034085$

58. Walter A, Suenderhauf C, Harrisberger F, Lenz C, Smieskova R, Chung Y, et al. Hippocampal volume in subjects at clinical high-risk for psychosis: a systematic review and meta-analysis. Neurosci Biobehav Rev. (2016) 71:68090. doi: 10.1016/j.neubiorev.2016.10.007

59. Pantelis C, Velakoulis D, McGorry P, Wood S, Suckling J, Phillips L, et al. Neuroanatomical abnormalities before and after onset of psychosis: a cross-sectional and longitudinal MRI comparison. Lancet. (2003) 361:281-8. doi: 10.1016/S0140-6736(03)12323-9

60. Giuliani NR, Calhoun VD, Pearlson GD, Francis A, Buchanan RW. Voxelbased morphometry versus region of interest: a comparison of two methods for analyzing gray matter differences in schizophrenia. Schizophr Res. (2005) 74:135-47. doi: 10.1016/j.schres.2004.08.019

61. Fusar-Poli P, Borgwardt S, Crescini A, Deste G, Kempton MJ, Lawrie S, et al. Neuroanatomy of vulnerability to psychosis: a voxelbased meta-analysis. Neurosci Biobehav Rev. (2011) 35:1175-85. doi: 10.1016/j.neubiorev.2010.12.005

62. Voevodskaya O, Simmons A, Nordenskjöld R, Kullberg J, Ahlström H, Lind $\mathrm{L}$, et al. The effects of intracranial volume adjustment approaches on multiple regional MRI volumes in healthy aging and Alzheimer's disease. Front Aging Neurosci. (2014) 6:264. doi: 10.3389/fnagi.2014.00264

63. Hedges L, Olkin I. Overlap between treatment and control distributions as an effect size measure in experiments. Psychol Methods. (2016) 21:61-8. doi: $10.1037 /$ met0000042

64. DerSimonian R, Laird N. Meta-analysis in clinical trials. Control Clin Trials. (1986) 7:177-88. doi: 10.1016/0197-2456(86)90046-2
65. Schwarzer G, Carpenter J, Rücker G. Metaanalysis With R. Berlin: Springer (2015).

66. Hu S, Coupé P, Pruessner JC, Collins D. Appearance-based modeling for segmentation of hippocampus and amygdala using multi-contrast MR imaging. NeuroImage. (2011) 58:549-59. doi: 10.1016/j.neuroimage.2011.06.054

67. Patenaude B, Smith S, Kennedy D, Jenkinson M. A Bayesian model of shape and appearance for subcortical brain segmentation. NeuroImage. (2011) 56:907-22. doi: 10.1016/j.neuroimage.2011.02.046

68. McHugo M, Armstrong K, Roeske MJ, Woodward N, Blackford J, Heckers S. Hippocampal volume in early psychosis: a 2-year longitudinal study. Transl Psychiatry. (2020) 10:306. doi: 10.1038/s41398-020-00985-1

69. Macdonald KE, Leung KK, Bartlett JW, Blair M, Malone I, Barnes J, et al. Automated template-based hippocampal segmentations from MRI: the effects of 1.5T or 3T field strength on accuracy. Neuroinformatics. (2014) 12:405-12. doi: 10.1007/s12021-013-9217-y

70. Lötjönen J, Wolz R, Koikkalainen J, Julkunen V, Thurfjell L, Lundqvist $\mathrm{R}$, et al. Fast and robust extraction of hippocampus from MR images for diagnostics of Alzheimer's disease. Neuroimage. (2011) 56:185-96. doi: 10.1016/j.neuroimage.2011.01.062

71. Blessing E, Murty V, Zeng B, Wang J, Davach L, Goff D. Anterior hippocampal-cortical functional connectivity distinguishes antipsychotic naïve first-episode psychosis patients from controls and may predict response to second-generation antipsychotic treatment. Schizophr Bull. (2020) 46:60889. doi: $10.1093 /$ schbul/sbz076

72. Jones SR, Fernyhough C. A new look at the neural diathesis-stress model of schizophrenia: the primacy of social-evaluative and uncontrollable situations. Schizophr Bull. (2007) 33:1171-7. doi: 10.1093/schbul/sbl058

Conflict of Interest: The authors declare that the research was conducted in the absence of any commercial or financial relationships that could be construed as a potential conflict of interest.

Copyright $\odot 2021$ Hinney, Walter, Aghlmandi, Andreou and Borgwardt. This is an open-access article distributed under the terms of the Creative Commons Attribution License (CC BY). The use, distribution or reproduction in other forums is permitted, provided the original author(s) and the copyright owner(s) are credited and that the original publication in this journal is cited, in accordance with accepted academic practice. No use, distribution or reproduction is permitted which does not comply with these terms. 